\title{
Clinicoradiological Features of Pulmonary Tuberculosis with Interstitial Pneumonia
}

\author{
Kazumasa Ogawa ${ }^{1}$, Atsuko Kurosaki ${ }^{2}$, Atsushi Miyamoto ${ }^{1}$, Yui Takahashi ${ }^{1}$, Kyoko Murase ${ }^{1}$, \\ Shigeo Hanada ${ }^{1}$, Hironori Uruga ${ }^{1}$, Hisashi Takaya ${ }^{1}$, Nasa Morokawa ${ }^{1}$ and Kazuma Kishi ${ }^{1,3}$
}

\begin{abstract}
:
Objective Although rare, pulmonary tuberculosis occasionally develops in patients with interstitial pneumonia (IP). In this study, we aimed to evaluate the clinicoradiological features of pulmonary tuberculosis associated with IP.

Methods In this retrospective, observational, single-center study, the medical charts, high-resolution computed tomography (HRCT) findings, and bacteriological test results of patients with IP who also tested positive for Mycobacterium tuberculosis were reviewed.

Patients The study included 20 patients with IP out of 329 who tested positive for M. tuberculosis in sputum or bronchoalveolar lavage fluid cultures at Toranomon Hospital between January 2006 and December 2017.

Results The HRCT patterns were usual interstitial pneumonia (UIP) in 11 patients and non-UIP in 9 patients. Consolidations $(80 \%)$ were the most frequent HRCT findings, followed by cavities $(60 \%)$ and nodules $(45 \%)$, which are generally characteristic of pulmonary tuberculosis. Consolidations often developed in relation to fibrotic or emphysematous lesions. Tuberculosis lesions could not be identified in one patient. All patients were treated with anti-tuberculosis drugs according to WHO guidelines, and 13 patients achieved a WHO category of "Treatment success." No patient died of tuberculosis, and the median survival time for the 20 patients was 1,196 days.

Conclusion Although the HRCT findings for pulmonary tuberculosis associated with IP are atypical, appropriate tuberculosis treatments can lead to favorable outcomes.
\end{abstract}

Key words: bronchoalveolar lavage fluid, interstitial lung diseases, sputum, tomography, tuberculosis

(Intern Med 58: 2443-2449, 2019)

(DOI: 10.2169/internalmedicine.2341-18)

\section{Introduction}

Interstitial pneumonia (IP) is a chronic, progressive fibrosing lung disease that manifests as non-segmental shadows in the lungs on high-resolution computed tomography (HRCT) (1). Pulmonary tuberculosis (TB) is a common cause of chronic lung infections worldwide (2) and is known to occasionally develop in patients with IP (3-5). Several studies of patients with IP have reported a positive culture rate for TB of 4.4-6.2\% (3-5), which is 4- to 5-fold higher than that for the general population.
The typical HRCT findings of TB include centrilobular nodules, a tree-in-bud appearance, calcifications, or cavity lesions (6-8). A few studies regarding mycobacterial infection with IP have highlighted atypical HRCT findings due to destruction of the lung parenchyma $(4,9)$. It can be difficult to diagnose TB based on HRCT findings in patients with pre-existing lung abnormalities, such as IP; notably, HRCT findings for TB patients with IP have not been sufficiently studied. Chung et al. (4) reported that, among 9 TB patients with idiopathic pulmonary fibrosis (IPF), the most common HRCT findings of TB were subpleural nodules and lobar or segmental consolidations in the lower lobes. Typical find-

${ }^{1}$ Department of Respiratory Medicine, Respiratory Center, Toranomon Hospital, Japan, ${ }^{2}$ Department of Diagnostic Radiology, Japan AntiTuberculosis Association, Fukujuji Hospital, Japan and ${ }^{3}$ Okinaka Memorial Institute for Medical Research, Japan

Received: November 4, 2018; Accepted: March 3, 2019; Advance Publication by J-STAGE: May 22, 2019

Correspondence to Dr. Kazumasa Ogawa, kaz_sap_tok@yahoo.co.jp 
ings, such as centrilobular nodules or tree-in-bud appearance, were infrequently seen in those patients. However, the number of patients was limited, and no other reports have described HRCT findings in TB patients with IP.

The clinical outcomes of TB with IP have been assessed in rather small populations (5). TB patients are typically treated according to the recommendations of the World Health Organization (WHO) guidelines (10); however, whether or not these standard therapies should be adapted to TB patients with IP is not yet clear. The possibility of developing acute exacerbation (AE) of IP by TB or TB treatment is a concern because AE of IP typically results in a high mortality rate; moreover, effective treatments have not been established (11). However, no reports on AE of IP in patients with TB have been published.

In this study, we aimed to reveal the clinical and HRCT characteristics of TB associated with IP. The findings are expected to promote the appropriate examination of these patients by clinicians.

\section{Materials and Methods}

The records of 329 patients admitted to Toranomon Hospital who tested positive for Mycobacterium tuberculosis in sputum or bronchoalveolar lavage fluid cultures between January 2006 and December 2017 were reviewed. Among these 329 patients, 20 consecutive patients who also presented with IP were evaluated. Medical charts, laboratory data, pulmonary function test results, HRCT findings, and results from bacterial tests were reviewed.

Patients were diagnosed with IP on the basis of official diagnostic recommendations of the American Thoracic Society/European Respiratory Society/Japanese Respiratory Society/Latin American Thoracic Association (1). The HRCT patterns of IP and the following features of TB were independently evaluated by one thoracic radiologist and two pulmonologists; the final diagnosis was determined by consensus regarding consolidations, cavities, nodules, calcifications, and thickening of the affected bronchial wall. The sites of the TB lesions were also assessed with regard to the association with fibrotic lesions and emphysematous lesions.

The most recent laboratory data and pulmonary function test results available prior to the definitive diagnosis of TB were reviewed. The severity of IP was calculated using a multidimensional index and staging system for IPF: the Gender-Age-Physiology (GAP) index (12). The GAP index is used globally to determine the severity of IPF using information regarding patients' gender, age, and results of pulmonary function tests. The GAP index ranges from 0 (most mild) to 8 (most severe). We adapted the GAP index for patients whose IP was not IPF in this study.

Regarding the estimated duration from the infection of TB until diagnosis of $\mathrm{TB}$, the timing of infection was determined using the earlier of the following events: 1) the point at which new abnormal shadows, caused by TB, were found in the lungs; 2) the point at which patients developed new symptoms due to TB.

All TB patients were treated according to the recommendations of the WHO guidelines (10). Treatment outcomes were also assessed using this guideline (10), which defines six per-patient clinical outcomes and one group outcome.

1) Cured: a TB patient with confirmed $\mathrm{TB}$ at the start of treatment who showed smear or culture negativity in the last month of treatment and on at least one previous occasion.

2) Treatment completed: a TB patient who completed treatment without evidence of sputum smear or culture negativity in the last month of treatment and on at least one previous occasion, without evidence of treatment failure; this may be because the relevant tests were not performed or because the results were unavailable.

3) Treatment failed: a TB patient whose sputum smear or culture remained positive at the fifth month or later during treatment.

4) Died: a TB patient who died for any reason before or during treatment.

5) Lost to follow-up: a TB patient who did not start treatment or whose treatment was interrupted for $\geq 2$ consecutive months.

6) Not evaluated: a TB patient for whom no treatment outcome was assigned, including patients transferred to another treatment unit and those for whom the treatment outcome was unknown.

7) Treatment success: all patients with the "Cured" and "Treatment completed" outcomes.

Adverse events were evaluated using the Common Terminology Criteria for Adverse Events ver. 4.0.

The survival time was analyzed with the Kaplan-Meier method. All statistical analyses were performed using the Statistical Package for the Social Sciences (SPSS) software program (version 18.0, SPSS, Chicago, USA).

This study was approved by the institutional review board of Toranomon Hospital, Tokyo, Japan (No. 823). All study participants provided their informed consent through an optout process.

\section{Results}

The prevalence of IP among the TB patients in this study was $6.1 \%$ (20/329). The characteristics of these 20 patients are presented in Table 1 . There were 17 men and 3 women with a median age of 74.5 years. IP was idiopathic in 14 patients. Four patients had IP related to connective tissue disease, one had chronic hypersensitivity pneumonia, and one had drug-induced IP. HRCT showed usual interstitial pneumonia (UIP) patterns in 11 patients and non-UIP patterns in 9. Fourteen patients had pulmonary emphysema. Many patients had additional complications, including malignancies in nine, diabetes mellitus in six, and liver diseases in six. Six patients were taking steroids, two were receiving immunosuppressants, and one was being administered a biological product. The median albumin, white blood cell, Creactive protein, Krebs von den Lugen-6, surfactant protein 
Table 1. Characteristics of Patients with Tuberculosis and Interstitial Pneumonia.

\begin{tabular}{|c|c|c|}
\hline \multicolumn{3}{|l|}{ Characteristics $(\mathrm{n}=20)$} \\
\hline Age & Median (range), years & $74.5(64-89)$ \\
\hline Sex & Male/Female (n) & $17 / 3$ \\
\hline Smoking status & Never smoker/Current or former smoker (n) & $3 / 17$ \\
\hline Brinkman index & Median (range) & $860(0-2,280)$ \\
\hline Etiology of IP & IIPs/Others (n) & $14 / 6$ \\
\hline HRCT patterns of IP & UIP pattern/non-UIP pattern (n) & $11 / 9$ \\
\hline Pulmonary emphysema & $+/-(\mathrm{n})$ & $14 / 6$ \\
\hline \multirow[t]{3}{*}{ Complications } & Malignancy (n) & 9 \\
\hline & Diabetes mellitus (n) & 6 \\
\hline & Liver diseases (n) & 6 \\
\hline \multirow[t]{3}{*}{ Use of immunosuppressants } & Steroid $(n)$ & 6 \\
\hline & Immunosuppressant (n) & 2 \\
\hline & Biological product (n) & 1 \\
\hline \multirow[t]{6}{*}{ Laboratory data } & Median Alb (g/dL) (range) & $2.9(1.8-3.9)$ \\
\hline & Median WBC $(/ \mu \mathrm{L})$ (range) & $7,750(3,000-17,100)$ \\
\hline & Median CRP (mg/dL) (range) & $5.1(0-17.5)$ \\
\hline & Median KL-6 (U/mL) (range) & $988(312-3,191)$ \\
\hline & Median SP-D (ng/mL) (range) & $153.5(30.7-993)$ \\
\hline & Median SP-A (ng/mL) (range) & $83.1(40.9-235.9)$ \\
\hline \multirow[t]{4}{*}{ Pulmonary function test } & Median FVC (mL) (range) & $2,095(1,260-4,050)$ \\
\hline & Median FVC, $\%$ predicted (range) & $75.1(37-128)$ \\
\hline & Median $\operatorname{FEV}_{1.0}(\%)$ (range) & $84.9(62.3-97.4)$ \\
\hline & Median $\mathrm{DL}_{\mathrm{co}}, \%$ predicted (range) & $51.5(17-110)$ \\
\hline GAP index & Median (range) & $4(2-6)$ \\
\hline
\end{tabular}

IP: interstitial pneumonia, IIPs: idiopathic interstitial pneumonias, HRCT: high-resolution computed tomography, UIP: usual interstitial pneumonia, Alb: albumin, WBC: white blood cells, CRP: C reactive protein, KL-6: Krebs von den Lungen-6, SP-D: surfactant protein D, SP-A: surfactant protein A, FVC: forced vital capacity, FEV 1.0 $_{\text {: }}$ forced expiratory volume 1.0 (s) percent, DLCO: diffusing capacity for carbon monoxide, GAP: Gender-AgePhysiology

$\mathrm{D}$, and surfactant protein A levels were $2.9 \mathrm{~g} / \mathrm{dL}, 7,750 \mathrm{U} /$ $\mu \mathrm{L}, 5.1 \mathrm{mg} / \mathrm{dL}, 988 \mathrm{U} / \mathrm{mL}, 153.5 \mathrm{ng} / \mathrm{mL}$, and $83.1 \mathrm{ng} / \mathrm{mL}$, respectively. Pulmonary function tests were performed for 12 patients. The median forced vital capacity (FVC), FVC percent predicted, and diffusing capacity for carbon monoxide percent predicted were $2,095 \mathrm{~mL}, 75.1 \%$, and $51.5 \%$, respectively. The median GAP index was calculated to be 4 .

Table 2 presents the clinical outcomes of TB in this study. The most common clinical symptoms were dyspnea (11 patients), cough (10 patients), fever ( 9 patients), sputum (7 patients), and weight loss (4 patients). Nine patients were diagnosed by a positive sputum smear, five by positive sputum culture, and six by bronchoalveolar lavage. Before a definitive diagnosis of TB of established, several patients were diagnosed with other conditions, including bacterial pneumonia in seven, fungal infection in three, interstitial pneumonia in two, and lung cancer in one. TB was the initial diagnosis for the remaining seven patients. With regard to the estimated duration from the onset of TB infection until the definitive diagnosis, seven patients were diagnosed within one month, nine were diagnosed within three months, and four were diagnosed three months or longer after TB infection.

Drug sensitivity tests revealed isoniazid (INH) resistance in one patient and levofloxacin resistance in one. Ten pa- tients were treated with INH, rifampicin (RFP), and ethambutol (EB), while eight were treated with INH, RFP, EB, and pyrazinamide (PZA). Increased liver enzymes comprised the most commonly observed adverse event. Grade 3 anorexia was also observed. There were no treatment discontinuations due to treatment-related adverse events. According to the WHO guidelines (10), eight patients were considered "Cured" and five achieved the "Treatment completed" outcome. Consequently, the "Treatment success" outcome was achieved in 13 patients. Three patients died, although the cause of death in all cases was not TB but other underlying diseases. The "Treatment failed" outcome was not observed for any patient. The survival time from the diagnosis of TB is presented in Fig. 1. The median survival time (MST) for the 20 patients was 1,196 days.

The HRCT findings for the 20 patients are summarized in Table 3. Infected TB lesions were identified in the upper lobes of $14(70 \%)$ patients, middle lobes of 5 (25\%) patients, and lower lobes of $13(65 \%)$ patients. Forty-five percent of the main TB lesions were identified in the upper lobes, $5 \%$ in the middle lobes, and $45 \%$ in the lower lobes. Among the 14 patients with pulmonary emphysema, main TB lesions were identified in the upper lobes in 7 (50\%) and lower lobes in $5(36 \%)$. Among the 6 patients without 
Table 2. Clinical Outcomes of Pulmonary Tuberculosis in This Study.

\begin{tabular}{|c|c|c|}
\hline \multirow[t]{5}{*}{ Symptoms (n) } & Dyspnea & 11 \\
\hline & Cough & 10 \\
\hline & Fever & 9 \\
\hline & Sputum & 7 \\
\hline & Weight loss & 4 \\
\hline \multirow[t]{3}{*}{ Diagnostic methods for TB (n) } & Sputum smear positivity & 9 \\
\hline & Sputum culture positivity & 5 \\
\hline & Bronchoalveolar lavage & 6 \\
\hline \multirow{5}{*}{$\begin{array}{l}\text { Primary diagnosis before definitive } \\
\text { diagnosis of TB (n) }\end{array}$} & Bacterial pneumonia & 7 \\
\hline & Fungal infection & 3 \\
\hline & Interstitial pneumonia & 2 \\
\hline & Lung cancer & 1 \\
\hline & Tuberculosis & 7 \\
\hline \multirow{3}{*}{$\begin{array}{l}\text { Estimated duration from TB infection } \\
\text { to definitive diagnosis of TB (n) }\end{array}$} & Within 1 month & 7 \\
\hline & Within 3 months & 9 \\
\hline & Over 3 months & 4 \\
\hline \multirow[t]{2}{*}{ Drug sensitivity examination (n) } & Isoniazid resistance & 1 \\
\hline & Levofloxacin resistance & 1 \\
\hline \multirow[t]{3}{*}{ Treatment drugs (n) } & Isoniazid/Rifampicin/Ethambutol/Pyrazinamide & 8 \\
\hline & Isoniazid/Rifampicin/Ethambutol & 10 \\
\hline & Others & 2 \\
\hline \multirow[t]{7}{*}{ Treatment outcomes $\ddagger(n)$} & Cured & 8 \\
\hline & Treatment completed & 5 \\
\hline & Treatment failed & 0 \\
\hline & Died & 3 \\
\hline & Lost to follow-up & 1 \\
\hline & Not evaluated & 3 \\
\hline & Treatment success & 13 \\
\hline
\end{tabular}

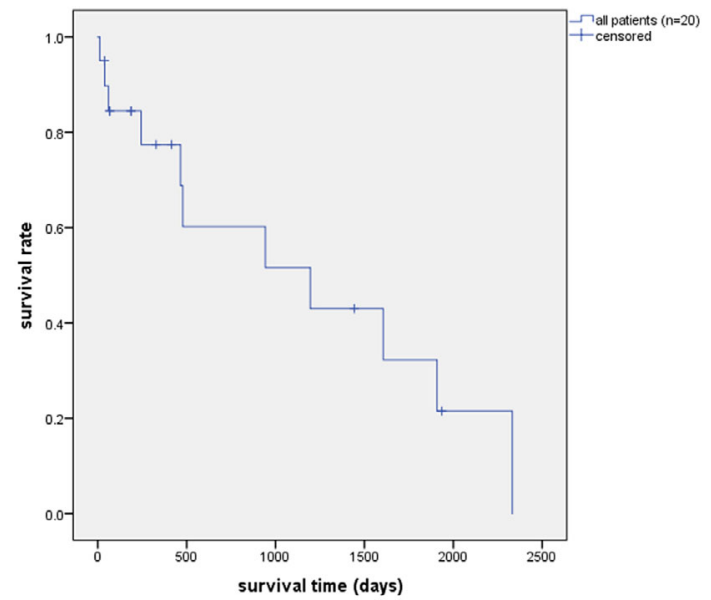

Figure 1. Survival curve showing the survival time from the diagnosis of tuberculosis in patients with interstitial pneumonia.

emphysema, 67\% exhibited their main TB lesions in lower lobes. Consolidations were the most frequent $\mathrm{CT}$ findings in this study (16 patients, 80\%), followed by cavities (12 patients, $60 \%$ ) and nodules (9 patients, $45 \%$ ), which are generally characteristic of TB. Consolidations often developed in relation to fibrotic or emphysematous lesions without centrilobular nodules (Fig. 2). Consolidations amid honeycomb cysts were found in five patients with the UIP pattern of IP. These consolidations appeared non-segmental because of destruction of the lung parenchyma.

\section{Discussion}

Several studies have reported a high prevalence of TB in patients with IP, which may be due to a number of factors, such as the administration of steroids and/or immunosuppressants, impaired local innate immune defense due to reduced ciliary clearance, and destruction of the anatomical structures of the lung parenchyma (3-5). However, thus far, the clinicoradiological features of TB associated with IP have not been well studied.

Few studies have evaluated the efficacy and safety of TB treatments for patients with TB associated with IP. Park et al. reported that approximately two-thirds of patients with TB and IPF were cured, while the remaining patients died of TB progression (5). Some patients in the present study developed anorexia or presented with increased liver enzymes. However, no serious adverse events developed, such as AE of IP. Although three patients died before the comple- 
Table 3. HRCT Findings for Patients with Pulmonary Tuberculosis and Interstitial Pneumonia.

\begin{tabular}{|c|c|c|c|}
\hline & $\begin{array}{l}\text { All patients } \\
(\mathrm{n}=20)\end{array}$ & $\begin{array}{l}\text { Patients with } \\
\text { UIP pattern } \\
(\mathrm{n}=11)\end{array}$ & $\begin{array}{c}\text { Patients with } \\
\text { non-UIP pattern } \\
(\mathrm{n}=9)\end{array}$ \\
\hline \multicolumn{4}{|l|}{ Infection site } \\
\hline Upper lobe & $14(70 \%)$ & $9(82 \%)$ & $5(56 \%)$ \\
\hline Middle lobe & $5(25 \%)$ & $1(9 \%)$ & $4(44 \%)$ \\
\hline Lower lobe & $13(65 \%)$ & $6(55 \%)$ & $7(78 \%)$ \\
\hline \multicolumn{4}{|l|}{ Main site of infection } \\
\hline Upper lobe & $9(45 \%)$ & $6(55 \%)$ & $3(33 \%)$ \\
\hline Middle lobe & $1(5 \%)$ & $0(0 \%)$ & $1(11 \%)$ \\
\hline Lower lobe & $9(45 \%)$ & $5(45 \%)$ & $4(44 \%)$ \\
\hline \multicolumn{4}{|l|}{ CT findings $\stackrel{\ddagger}{\ddagger}$} \\
\hline Consolidation & $16(80 \%)$ & $9(82 \%)$ & $7(78 \%)$ \\
\hline Segmental consolidation & $10(50 \%)$ & $4(36 \%)$ & $6(67 \%)$ \\
\hline Non-segmental consolidation & $8(40 \%)$ & $7(64 \%)$ & $1(11 \%)$ \\
\hline Consolidation related to emphysematous lesions & $5(25 \%)$ & $3(27 \%)$ & $2(22 \%)$ \\
\hline Consolidation in the midst of honeycomb cysts & $6(30 \%)$ & $5(45 \%)$ & $1(11 \%)$ \\
\hline Cavity & $12(60 \%)$ & $6(55 \%)$ & $6(67 \%)$ \\
\hline Nodule & $9(45 \%)$ & $4(36 \%)$ & $5(56 \%)$ \\
\hline Centrilobular nodule & $7(35 \%)$ & $4(36 \%)$ & $3(33 \%)$ \\
\hline Random nodule & $1(5 \%)$ & $0(0 \%)$ & $1(11 \%)$ \\
\hline Tree-in-bud appearance & $4(20 \%)$ & $2(18 \%)$ & $2(22 \%)$ \\
\hline Calcification & $7(35 \%)$ & $5(45 \%)$ & $2(22 \%)$ \\
\hline \multicolumn{4}{|l|}{ Thickening of affected } \\
\hline bronchial wall & $3(15 \%)$ & $2(18 \%)$ & $1(11 \%)$ \\
\hline Unidentifiable & $1(5 \%)$ & $1(9 \%)$ & $0(0 \%)$ \\
\hline
\end{tabular}

¥There was a degree of overlap.

HRCT: high-resolution computed tomography, UIP: usual interstitial pneumonia

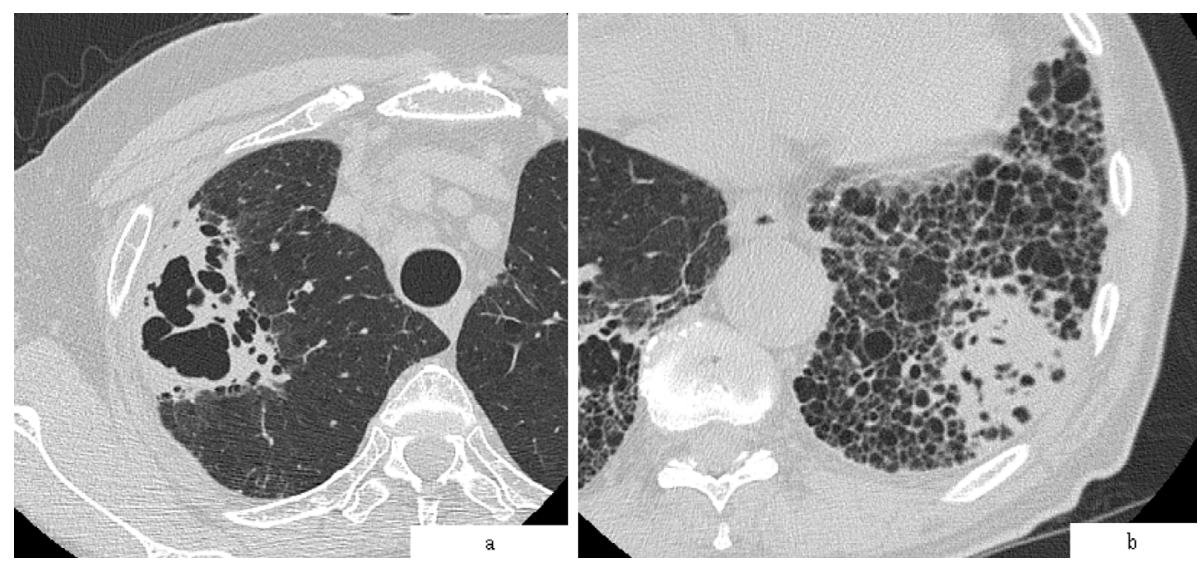

Figure 2. Major high-resolution computed tomography findings for patients with tuberculosis and interstitial pneumonia. a) Consolidation related to emphysematous lesions that do not accompany centrilobular nodules in the upper lobe. b) Consolidation amid honeycomb cysts in the lower lobe, without centrilobular nodules.

tion of TB treatment because of worsening of their underlying illness, no patients died of TB progression. Furthermore, no patient achieved the "Treatment failed" outcome, according to the WHO guidelines. These findings indicate that appropriate treatment regimens for TB patients with IP can lead to favorable outcomes. The low frequency of multidrug-resistant TB in the present study may have contributed to these good outcomes. TB treatment with INH, RFP, and EB was chosen for 10 patients, while treatment with INH, RFP, EB, and PZA was chosen for 8 patients; these approaches resulted in respective "Treatment success" rates of $60 \%$ and $88 \%$. Reasons for the non-use of PZA were age $>80$ years old in 7 patients, cirrhosis of the liver in 1 patient, and unknown in 2 patients; relatively poor treatment 
outcomes may have been observed in patients who did not receive PZA. Although whether or not standard TB therapies should be adapted for TB patients with IP remains unclear, the results of the present study support the efficacy and safety of standard TB treatment for patients with IP.

Only $45 \%$ of main TB infection lesions were observed in the upper lobes of the lung in the present study; this contrasted with the more typical infection pattern for secondary $\mathrm{TB}$, which reportedly develops in the upper lobes at a frequency of $91 \%$ (7). The tendency of the main infected lesions in the lower lobes appeared similar between patients with the UIP pattern of IP and those with the non-UIP pattern of IP. This may occur because abnormal shadows tend to develop in the lower lobes in patients with the non-UIP pattern of IP, as well as those with the UIP pattern of IP, although patients with the non-UIP pattern of IP usually do not exhibit honeycomb changes.

Regarding emphysema, 14 patients exhibited pulmonary emphysema. The main infected site was in the upper lobes in $50 \%$ of patients with pulmonary emphysema, whereas it was in lower lobes in $67 \%$ of patients without emphysema. Impaired local immune defense and reduced ciliary clearance caused by emphysema might have resulted in these differences in frequency of TB in upper lobes, as emphysema is typically observed more frequently in the upper lobes than in the lower lobes.

Chung et al. (4) reported nine TB patients with IPF, for whom the most common HRCT findings for TB were subpleural nodules and lobar or segmental consolidations in the lower lobes. More typical TB findings, such as multifocal patchy consolidations or centrilobular nodules $(6,7)$, were infrequent in that study. Hwang et al. found lobar/segmental consolidations on the HRCT images of patients with nontuberculous mycobacterial infection and IP, whereas branching centrilobular nodules and traction bronchiectasis were common in the patients without IP (9). The absence of centrilobular nodules on HRCT images was also noted in a report of TB in patients with pulmonary emphysema (13). The present study also found an increased tendency for consolidations and a relatively low tendency for centrilobular nodules on HRCT images. Consolidations had developed in previously formed emphysematous lesions or honeycomb lungs. Airway-oriented lesions may not be identified in such patients because pulmonary acini, where mycobacterial infections typically develop first, are damaged by emphysema or fibrosis. Immunodeficiency is another possible explanation for these unusual radiological features. Similar to fungal infections (14), TB reportedly exhibits unusual radiological findings in the context of immunodeficiency $(15,16)$; the reduced ability of the immune system to control the bacterial infection might result in unusual radiological findings, such as the infrequent presence of cavities.

In total, 5 patients $(25 \%)$ in the present study exhibited only consolidations adjacent to or inside fibrotic lesions without cavities or centrilobular nodules, and 1 patient $(5 \%)$ had no apparent TB lesions, which complicated the defini- tive identification of possible lesions. Bacterial pneumonia was the most common initial diagnosis before the confirmation of TB infection, and these patients were primarily treated with antibiotics. The estimated duration from the onset of TB infection to the definitive diagnosis of TB was $>1$ month in 13 TB patients with IP. This is partially because of the abnormal HRCT findings of TB in patients with IP and partially because of the difficulty in noticing symptoms of TB, which are typically similar to the symptoms of IP. Despite the difficulties in diagnosing TB, appropriate TB treatment led to favorable outcomes. These results indicate the importance of testing for TB in patients with IP whose HRCT findings are not typical for TB.

This study has several limitations. First, it was a retrospective, single-center study with a small patient cohort, and some patients included in the study did not have a complete set of follow-up data. Second, the prevalence of TB was not evaluated in patients with IP. Finally, we did not directly compare TB patients with IP to those without IP. However, TB without IP is a common disease, and its clinicoradiological characteristics are well-known. We further analyzed 20 consecutive TB patients without IP and found that the main TB infected site was the upper lobes in $70 \%$ of patients and lower lobes in $30 \%$ of patients. Centrilobular nodules and tree-in-bud appearances were the most frequently observed HRCT findings (85\%), followed by cavities (40\%) and consolidations $(20 \%)$. These results are consistent with those of past reports $(6,7,9)$ supporting the unique characteristics of TB patients with IP in the present study. Additional studies are needed in this patient population for a more complete understanding of the implications of IP during the interpretation of HRCT images obtained for TB patients with IP.

In conclusion, consolidations, and not the characteristic TB-associated findings of cavities and nodules, were the most frequently observed HRCT findings in the TB patients with IP in the present study. Consolidations adjacent to or inside fibrotic or emphysematous lesions without centrilobular nodules or cavities complicated the TB diagnosis in these patients. Our findings suggest that TB should always be considered as a differential diagnosis when consolidations develop in patients with IP, as favorable outcomes can be achieved with appropriate treatment for patients with IP who develop TB.

\section{The authors state that they have no Conflict of Interest (COI).}

\section{References}

1. Raghu G, Collard HR, Egan JJ, et al. An official ATS/ERS/JRS/ ALAT statement: idiopathic pulmonary fibrosis: evidence-based guidelines for diagnosis and management. Am J Respir Crit Care Med 183: 788-824, 2011.

2. Kochi A. The global tuberculosis situation and the new control strategy of the World Health Organization. 1991. Bull World Health Organ 79: 71-75, 2001.

3. Shachor Y, Schindler D, Siegal A, Lieberman D, Mikulski Y, Bruderman I. Increased incidence of pulmonary tuberculosis in 
chronic interstitial lung disease. Thorax 44: 151-153, 1989.

4. Chung MJ, Goo JM, Im JG. Pulmonary tuberculosis in patients with idiopathic pulmonary fibrosis. Eur J Radiol 52: 175-179, 2004.

5. Park SW, Song JW, Shim TS, et al. Mycobacterial pulmonary infections in patients with idiopathic pulmonary fibrosis. J Korean Med Sci 27: 896-900, 2012.

6. Goo JM, Im JG. CT of tuberculosis and nontuberculous mycobacterial infections. Radiol Clin North Am 4073-4087, 2002

7. Woodring JH, Vandiviere HM, Fried AM, Dillon ML, Williams TD, Melvin IG. Update: the radiographic features of pulmonary tuberculosis. Am J Roentgenol 146: 497-506, 1986.

8. Park S, Hong YK, Joo SH, Choe KO, Cho SH. CT findings of pulmonary tuberculosis presenting as segmental consolidation. J Comput Assist Tomogr 23: 736-742, 1999.

9. Hwang HJ, Kim MY, Shim TS, Kim DS. Nontuberculous mycobacterial pulmonary infection in patients with idiopathic interstitial pneumonias: comparison with patients without idiopathic interstitial pneumonias. J Comput Assist Tomogr 38: 972-978, 2014.

10. World Health Organization. Treatment of tuberculosis: Guidelines. 4th ed. 2010.

11. Collard HR, Ryerson CJ, Corte TJ, et al. Acute exacerbations of idiopathic pulmonary fibrosis. Am J Respir Crit Care Med 176: 636-643, 2007.
12. Ley B, Ryerson CJ, Vittinghoff E, et al. A multidimensional index and staging system for idiopathic pulmonary fibrosis. Ann Intern Med 156: 684-691, 2012.

13. Jeon KN, Ha JY, Park MJ, et al. Pulmonary tuberculosis in patients with emphysema: computed tomography findings. J Comput Assist Tomogr 40: 912-916, 2016.

14. Visnegarwala F, Graviss EA, Lacke CE, et al. Acute respiratory failure associated with cryptococcosis in patients with AIDS: analysis of predictive factors. Clin Infect Dis 27: 1231-1237, 1998.

15. Kobashi Y, Mouri K, Yagi S, et al. Clinical features of immunocompromised and nonimmunocompromised patients with pulmonary tuberculosis. J Infect Chemother 13: 405-410, 2007.

16. Ikezoe J, Takeuchi N, Johkoh $\mathrm{T}$, et al. CT appearance of pulmonary tuberculosis in diabetic and immunocompromised patients: comparison with patients who had no underlying disease. Am J Roentgenol 159: 1175-1179, 1992.

The Internal Medicine is an Open Access journal distributed under the Creative Commons Attribution-NonCommercial-NoDerivatives 4.0 International License. To view the details of this license, please visit (https://creativecommons.org/licenses/ by-nc-nd/4.0/).

(C) 2019 The Japanese Society of Internal Medicine Intern Med 58: 2443-2449, 2019 